\title{
Supporting information for "Direct Laser Writing from Aqueous Precursors for Nano to Microscale Topographical Control, Integration, and Synthesis of Nanocrystalline Mixed Metal Oxides"
}

Cody Kindle ${ }^{1}$, Alexander Castonguay ${ }^{1}$, Shannon McGee ${ }^{1}$, John A. Tomko ${ }^{2}$, Patrick E. Hopkins ${ }^{2-4}$, and Lauren D. Zarzar*1,5

1. Department of Chemistry, The Pennsylvania State University, University Park, PA 16802

2. Department of Materials Science and Engineering, University of Virginia, Charlottesville, VA, 22904

3. Department of Mechanical and Aerospace Engineering, University of Virginia, Charlottesville, VA, 22904

4. Department of Physics, University of Virginia, Charlottesville, VA, 22904

5. Materials Research Institute, The Pennsylvania State University, University Park, PA 16802

*corresponding author, Idz4@psu.edu

\section{Supporting Videos}

Video $\mathrm{S} 1 . \mathrm{Mn}_{2} \mathrm{O}_{3}$ is laser deposited off of a platinum line using manganese nitrate as the precursor. Bubbles generated at the reaction site rapidly detach from the surface.

Video S2. $\mathrm{Cr}_{2} \mathrm{O}_{3}$ is laser deposited off of a platinum line using chromium nitrate as the precursor. Significant pinning and spreading of the bubble at the deposition site contributes to decreased resolution.

\section{Chemicals}

All chemicals are commercially available and used as received. $\mathrm{Al}\left(\mathrm{NO}_{3}\right)_{3} \cdot 9 \mathrm{H}_{2} \mathrm{O}$ reagent grade, $\mathrm{Co}\left(\mathrm{NO}_{3}\right)_{2} \cdot 6 \mathrm{H}_{2} \mathrm{O}$ reagent grade (Fisher Science); $\mathrm{TiOSO}_{4} \cdot \mathrm{xH}_{2} \mathrm{SO}_{4} \cdot \mathrm{yH}_{2} \mathrm{O}$ synthesis grade, $\mathrm{Zn}\left(\mathrm{NO}_{3}\right)_{2} \cdot 6 \mathrm{H}_{2} \mathrm{O}>99.0 \%, \mathrm{Na}_{3} \mathrm{IrCl} \cdot \mathrm{xH}_{2} \mathrm{O}, \mathrm{Mn}\left(\mathrm{NO}_{3}\right)_{2} 50 \%$ solution (Sigma Aldrich); $\mathrm{VOSO}_{4} \cdot \mathrm{xH}_{2} \mathrm{O} 99.9 \%, \mathrm{Cr}\left(\mathrm{NO}_{3}\right)_{3} \cdot 9 \mathrm{H}_{2} \mathrm{O} 98.5 \%, \mathrm{FeN}_{3} \mathrm{O}_{9} \cdot \mathrm{H}_{2} \mathrm{O} 98.0 \%, \mathrm{FeCl}_{2} \cdot 4 \mathrm{H}_{2} \mathrm{O} 98.0 \%, \mathrm{Ni}\left(\mathrm{NO}_{3}\right)_{2} \cdot 6 \mathrm{H}_{2} \mathrm{O} 98 \%, \mathrm{Y}\left(\mathrm{NO}_{3}\right)_{3} \cdot 6 \mathrm{H}_{2} \mathrm{O}$ 99\%, $\mathrm{ZrO}\left(\mathrm{NO}_{3}\right)_{2} \cdot \mathrm{xH}_{2} \mathrm{O} 99.95 \%,\left(\mathrm{NH}_{4}\right)_{6} \mathrm{Mo}_{7} \mathrm{O}_{24} \cdot 4 \mathrm{H}_{2} \mathrm{O} 99 \%,\left(\mathrm{NH}_{4}\right)_{3} \mathrm{RhCl}_{6} \cdot \mathrm{HH}_{2} \mathrm{O} 99.99 \%$, $\left(\mathrm{NH}_{4}\right)_{2} \mathrm{PdCl}_{6} 99.9 \%, \mathrm{AgNO}_{3} 99.9 \%, \mathrm{NH}_{4} \mathrm{ReO}_{4}$ 99+\%, $\mathrm{H}_{8} \mathrm{Cl}_{4} \mathrm{~N}_{2} \mathrm{Pt}$ 99.9\%, $\left(\mathrm{NH}_{4}\right) \mathrm{AuCl}_{4} \cdot \mathrm{xH}_{2} \mathrm{O} 99.99 \%$, $\left(\mathrm{NH}_{4}\right)_{2} \mathrm{RuCl}_{6} 99.99 \%$ (Alfa Aesar); $\mathrm{Cu}\left(\mathrm{NO}_{3}\right)_{2} \cdot 6 \mathrm{H}_{2} \mathrm{O},\left(\mathrm{NH}_{4}\right)_{3} \mathrm{Fe}\left(\mathrm{C}_{2} \mathrm{O}_{4}\right)_{3} \cdot 3 \mathrm{H}_{2} \mathrm{O} 98 \%$, $\mathrm{C}_{6} \mathrm{H}_{5} \mathrm{Na}_{3} \mathrm{O}_{7} 98 \%$ (Acros Organics). All metal and metal oxide precursor solutions were prepared by dissolving the precursor salts in reverse osmosis water at concentrations found in Table 1 in the primary text.

\section{Direct Laser Writing Optical Setup}

All depositions employed a Coherent Mira Optima 900-F titanium:sapphire laser operating at $760 \mathrm{~nm}$ in either femtosecond pulse mode (for initial deposition of platinum absorber) or continuous wave (for deposition of metal and metal oxide materials). The laser output was controlled using a half wave-plate/polarizing beam-splitter pair, with the half wave-plate controlled by a motorized rotation mount (Thorlabs K10CR1) which allowed for continuous tuning of the laser power during deposition. The laser focus was translated using a galvo scanner (Nutfield open frame head XLR8 - $10 \mathrm{~mm}$ clear aperture), which allowed oscillation of the laser spot as it entered a microscope objective (air 40x Nikon Plan Fluor objective with 0.60 NA, or a 60x Nikon Plan Fluor oil immersion objective with $1.4 \mathrm{NA}$ ) situated on an inverted microscope (ASI automated RAMM/ modular infinity microscope system with motorized stage). Lenses were used to collimate and expand the beam to fill the back of the microscope objective. Laser powers reported were measured at the back of the objective. A schematic of the laser writing setup is below. 


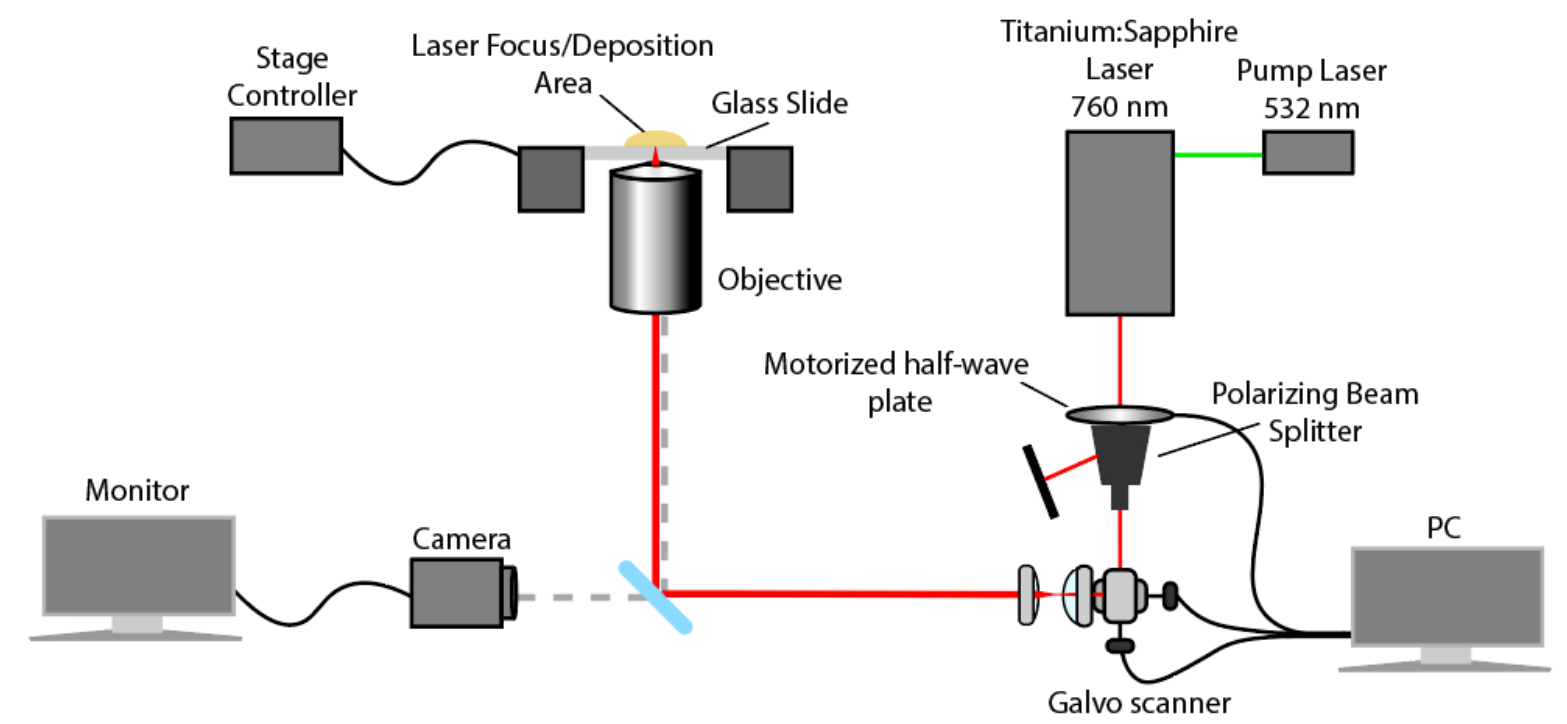

Scheme S1. Schematic representation of the direct laser writing optical setup, as described in detail in the Supporting Information section "Direct Laser Writing Optical Setup".

\section{Material Deposition}

The initial optical absorber typically used was platinum, deposited onto a borosilicate glass coverslip using a multiphoton reduction process as described elsewhere ${ }^{1}$. The platinum precursor was then washed off, and a new precursor was placed on the coverslip. The laser was focused through a microscope objective onto the platinum absorber to create the laser induced thermal voxel, thereby depositing material directly from the precursor fluid. Patterns were "drawn" with the laser by both scanning the laser and translating the microscope stage. To switch materials, the precursor fluid was rinsed off and replaced. In order to deposit materials in layers, the laser was rastered over the bottom-layer material with an increased power to promote the deposition of the second material on top. This could be repeated for multiple layers.

\section{Annealing}

The iron oxide sample in Figure 2 was annealed using a Thermolyne 48000 furnace with a ramp rate of $10{ }^{\circ} \mathrm{C} / \mathrm{min}$ to $450{ }^{\circ} \mathrm{C}$ for 4 hours with a cool down rate of $1^{\circ} \mathrm{C} / \mathrm{min}$. The sample was analyzed using X-ray diffraction (XRD) and Raman spectroscopy before and after annealing. Laser annealing with also conducted in-situ during Raman analysis with a $532 \mathrm{~nm}$ laser; spectra were collected at $1 \%$ power $(0.45 \mathrm{~mW})$ after annealing for 10 seconds each at increasingly higher power as detailed in Figure 2 . Exposure times longer than 10 seconds did not correspond to any additional changes in material structure.

\section{Characterization}

X-Ray Diffraction. XRD was performed using a PANalytical Xpert Pro MPD with a Cu source, a theta-theta goniometer, parallel beam optics, line focus in reflection mode, with a PIXcel 1D detector operating from 20-80 $2 \theta$ for a 15 minute scan using a multi-purpose stage.

Raman Spectroscopy. Raman spectroscopy was performed using a $532 \mathrm{~nm}$ laser with a maximum power at the sample of 45 $\mathrm{mW}$ (notch filter $\pm 10 \mathrm{~cm}^{-1}$ ), a back Illuminated detector (2048x512 pixel), a 100x objective (NA 0.9 WD $0.2 \mathrm{~mm}$ ), and $300 \mathrm{~g} / \mathrm{mm}$ grating (spectral resolution $\sim 4 \mathrm{~cm}^{-1} @ 532 \mathrm{~nm}$ ) using an input laser power range of $1 \%$ to $25 \%$ power.

Optical Profilometry. Profilometry was performed using a Zygo Nexview 3D profilometer with a 50x objective with a $2 x$ internal multiplier. Auto-light settings were used to properly image topography, with an internal masking and calibration used to set baselines of the glass background.

Scanning Electron Microscopy (SEM) and Energy Dispersity X-ray Spectroscopy (EDS). SEM images and EDS maps were taken on a FEI Nova NanoSEM 630 SEM with an accelerating voltage of $7 \mathrm{keV}$ for imaging and $15 \mathrm{keV}$ for EDS. Samples were coated with a $5 \mathrm{~nm}$ layer of iridium metal to prevent charge build-up. 
Cross Sectioning. Cross sectioning was done by first cutting excess glass to expose the sample using a Leica EM TPX. Polishing was then done with a $9 \mu \mathrm{m}$ diamond lapping foil to smooth the surface and prepare the sample for ion milling. The final ion milling was done with a Leica EM TIC $3 X$ for 4 hrs at $8 \mathrm{kV}$.

Measurement of Thermal Conductivity. To measure the thermal conductivity of the direct-write structures, we deposited an 80 $\mathrm{nm} \mathrm{Al} \mathrm{film} \mathrm{on} \mathrm{the} \mathrm{microstructures} \mathrm{via} \mathrm{electron} \mathrm{beam} \mathrm{evaporation} \mathrm{to} \mathrm{perform} \mathrm{time-domain} \mathrm{thermoreflectance} \mathrm{(TDTR)}$ measurements; details of the specific TDTR system has been discussed in-depth in previous works ${ }^{2-3}$. In brief, TDTR is a pumpprobe method in which a modulated laser pulse creates a heating event on the surface of a thin metal transducer. A second laser pulse, the probe beam, is temporally-delayed with a mechanical delay stage and interacts with the heated area; we measure the reflectivity of the probe beam as a function of delay time between the two pulses, thus obtaining a rate of thermal dissipation into the underlying substrate. This curve is then fit with a thermal model ${ }^{4}$ to obtain the thermal properties of the material system of interest.

The measured values for thermal conductivity as well as values for the volumetric heat capacity, as obtained in reference ${ }^{5}$ are shown in Table S3. We note that the sample surface is more rough than typical TDTR measurements and the underlying structure degrades at relatively low power densities, leading to higher error than typically found in TDTR experiments. Further, as we use fully-dense, bulk material values for the volumetric heat capacity in our thermal model, any changes in the sample density will lead to a change in the measured thermal conductivity. Nonetheless, the lack of trend between line-width and thermal conductivity of the direct write structure is quite clear.

The thermal conductivity of a crystalline $\mathrm{Fe}_{2} \mathrm{O}_{3}$ (hematite) was extrapolated to $6.4 \mathrm{~W} \mathrm{~m}^{-1} \mathrm{~K}^{-1}$ at room temperature, based on previously reported data ${ }^{6}$. As the deposited iron oxide in this work is known to be amorphous, a marked decrease in thermal conductivity would be expected. Literature values for the thermal conductivity of copper oxide are quite wide-spread and are recognized to be highly phase-dependent. For example, $\mathrm{Cu}_{2} \mathrm{O}$ has a reported thermal conductivity of $\sim 5 \mathrm{~W} \mathrm{~m}^{-1} \mathrm{~K}^{-1}$, with $a b$ initio calculations predicting even lower values ${ }^{8}$, whereas $\mathrm{CuO}$ has been assumed to have values as high of $69 \mathrm{~W} \mathrm{~m}^{-1} \mathrm{~K}^{-1}$ or as low as $20 \mathrm{~W} \mathrm{~m}^{-1} \mathrm{~K}^{-1} \cdot{ }^{8-11}$. It is quite possible that these variations are due to physical differences in measured samples, and thus contribute to the large error found in the measured thermal conductivity of $\mathrm{CuO}$ in this work. Similar issues in comparing our measured thermal conductivity values to reported literature values for $\mathrm{Cr}_{2} \mathrm{O}_{3}$ and $\mathrm{Co}_{3} \mathrm{O}_{4}$, as both lack consistent values. For example, Hay et al. report a value of $\sim 5 \mathrm{~W} \mathrm{~m}^{-1} \mathrm{~K}^{-1}$ for $\mathrm{Cr}_{2} \mathrm{O}_{3}$ coatings ${ }^{12}$, whereas Marinelli et al. measure a thermal conductivity of $\sim 13 \mathrm{~W} \mathrm{~m}^{-1} \mathrm{~K}^{-1}$ for bulk $\mathrm{Cr}_{2} \mathrm{O}_{3}$ at room temperature ${ }^{13}$.

\begin{tabular}{|c|c|c|}
\hline Material & Line width $(\boldsymbol{\mu m})$ & Laser power $(\mathbf{m W})$ \\
\hline $\mathrm{Mn}_{2} \mathrm{O}_{3}$ & 2.5 & 23 \\
\hline $\mathrm{CuO}$ & 2.5 & 32 \\
\hline $\mathrm{NiO}$ & 5.5 & 53 \\
\hline $\mathrm{Cr}_{2} \mathrm{O}_{3}$ & 8 & 37 \\
\hline $\mathrm{Fe}_{2} \mathrm{O}_{3}$ & 14.5 & 53 \\
\hline $\mathrm{CO}_{3} \mathrm{O}_{4}$ & 2.5 & 23 \\
\hline $\mathrm{Pt}$ & 2.5 & 47 \\
\hline
\end{tabular}

Table S1. Minimum line widths achieved for a range of materials using a 60x, 1.4 N.A. objective at the stated laser powers as measured at the back of the objective. Lines were deposited by rastering the laser using a galvoscanner in a 10 micron line at a rate of 5 passes/s. The stage was manually translated along the direction of the scan to induce material deposition. The galvo scanning was used in addition to the stage translation in order to promote bubble de-pinning at the leading edge of deposition. Laser power was modulated to find minimum achievable line widths. Precursors used were the same as given in Table 1. 


\begin{tabular}{|c|c|}
\hline Material & Line Width $(\boldsymbol{\mu m})$ \\
\hline $\mathrm{Cr}_{2} \mathrm{O}_{3}$ & 8.1 \\
\hline $\mathrm{Mn}_{2} \mathrm{O}_{3}$ & 8.1 \\
\hline $\mathrm{CuO}$ & 2.5 \\
\hline $\mathrm{NiO}$ & 5.4 \\
\hline $\mathrm{Co}_{3} \mathrm{O}_{4}$ & 8.3 \\
\hline $\mathrm{Fe}_{2} \mathrm{O}_{3}$ & 14.5 \\
\hline
\end{tabular}

Table S2. Line widths for a range of materials deposited under the same laser deposition conditions. Materials were deposited by rastering the laser in a 10 micron line at rate of 5 passes/s and manually translating the stage in the direction of the scan. All samples deposited using a $60 x$, N.A. 1.4 objective at a power of $53 \mathrm{~mW}$ as measured at the back of the objective. Precursors used were the same as given in Table 1.

\begin{tabular}{|c|c|c|c|c|}
\hline Material & Specific Heat (cal $\mathrm{g}^{-1} \mathrm{~K}^{-1}$ ) & Density $\left(\mathrm{g} \mathrm{cm}^{-3}\right)$ & $\begin{array}{l}\text { Thermal Conductivity } \\
\qquad\left(\mathrm{W} \mathrm{m}^{-1} \mathrm{~K}^{-1}\right)\end{array}$ & $\begin{array}{c}\text { Error in Thermal } \\
\text { Conductivity }\end{array}$ \\
\hline $\mathrm{Co}_{3} \mathrm{O}_{4}$ & 0.125 & 6.11 & 0.8 & 0.3 \\
\hline $\mathrm{CuO}$ & 0.125 & 6.31 & 26.6 & 10 \\
\hline $\mathrm{Cr}_{2} \mathrm{O}_{3}$ & 0.175 & 5.22 & 1.54 & 0.5 \\
\hline $\mathrm{Fe}_{2} \mathrm{O}_{3}$ & 0.15 & 5.24 & 1.7407 & 0.5 \\
\hline
\end{tabular}

Table S3. Data for volumetric heat capacity (from reference ${ }^{5}$ ) and measured thermal conductivity of selected oxides. We do not observe correlation of thermal conductivity with minimum line width trends. 

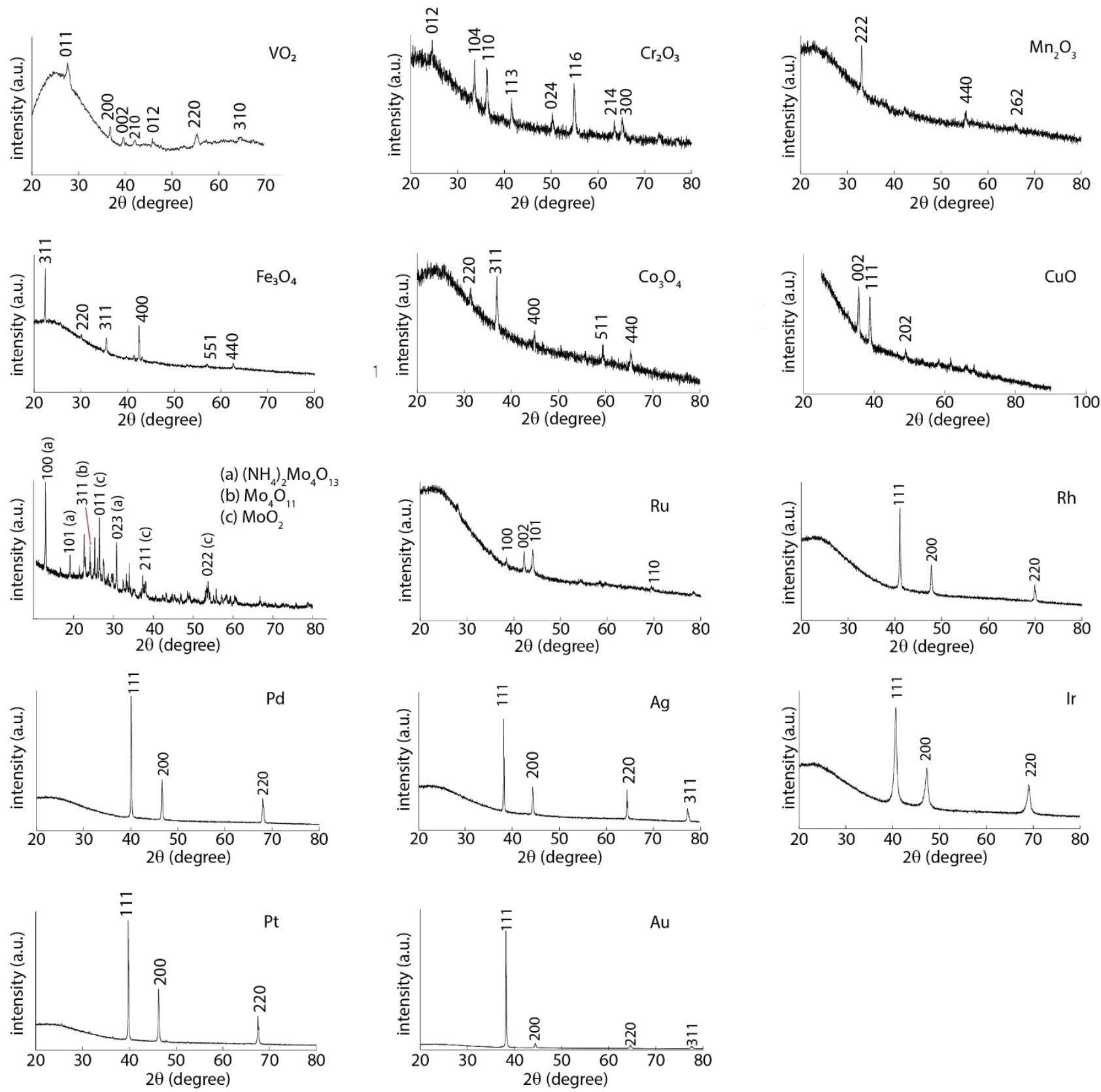

Figure S1. XRD data used to identify compounds listed in Table 1 in the main text. 

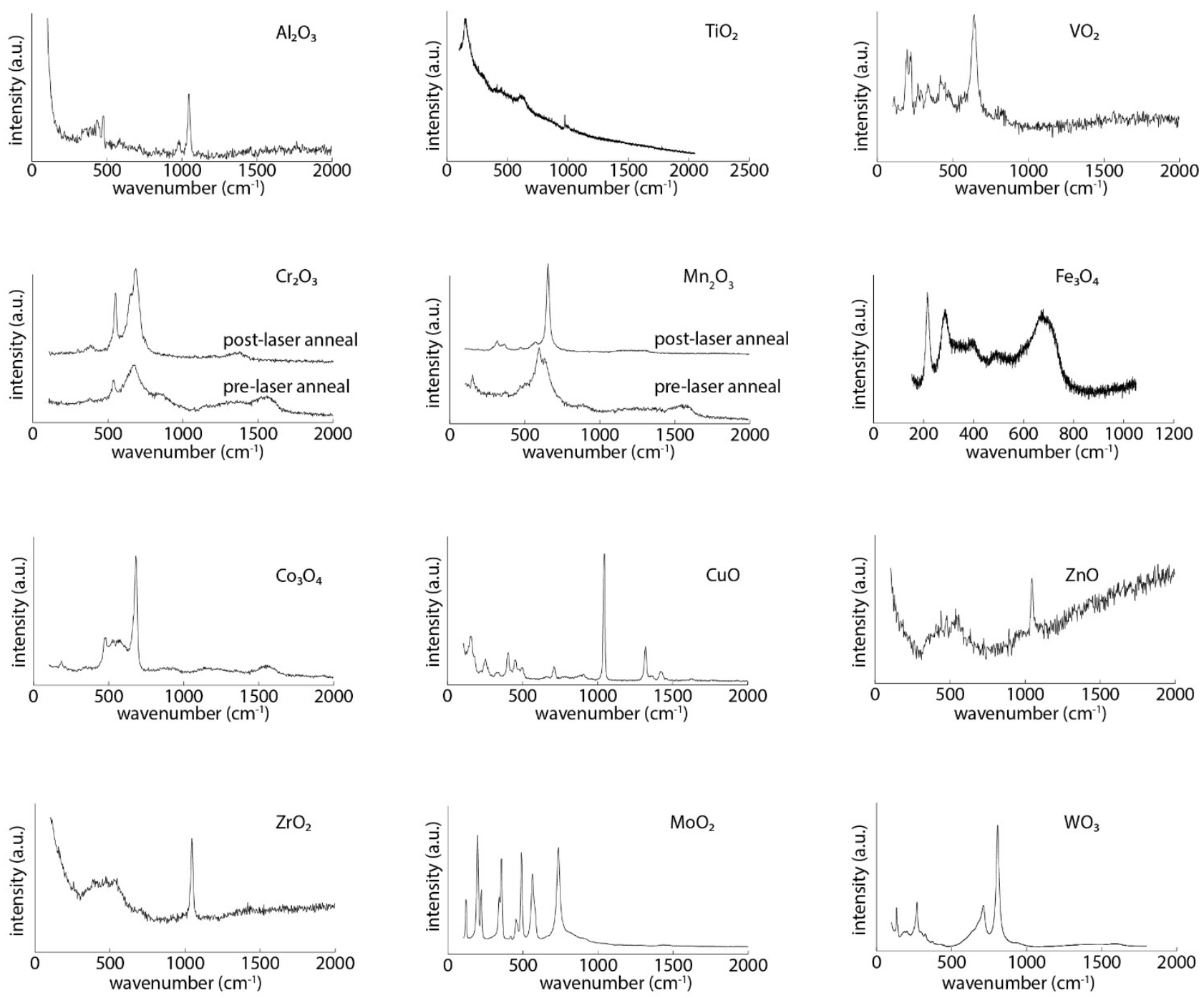

Figure S2. Raman spectroscopy data used for identification of compounds listed in Table 1 of the main text. For the $\mathrm{Cr}_{2} \mathrm{O}_{3}$ and $\mathrm{Mn}_{2} \mathrm{O}_{3}$ in-situ laser annealing during Raman analysis using the $532 \mathrm{~nm}$ laser was conducted, similar to the experiment described in Figure 2, and the pre- and post-anneal spectra are given for comparison.

\section{References}

1. Zarzar, L. D.; Swartzentruber, B. S.; Donovan, B. F.; Hopkins, P. E.; Kaehr, B., Using laser-induced thermal voxels to pattern diverse materials at the solid-liquid interface. ACS Applied Materials \& Interfaces 2016, 8 (33), 21134-21139.

2. Hopkins, P. E.; Duda, J. C.; Kaehr, B.; Wang Zhou, X.; Peter Yang, C.-Y.; Jones, R. E., Ultrafast and steadystate laser heating effects on electron relaxation and phonon coupling mechanisms in thin gold films. Applied Physics Letters 2013, 103 (21), 211910.

3. Hopkins, P. E.; Serrano, J. R.; Phinney, L. M.; Kearney, S. P.; Grasser, T. W.; Harris, C. T., Criteria for CrossPlane Dominated Thermal Transport in Multilayer Thin Film Systems During Modulated Laser Heating. Journal of Heat Transfer 2010, 132 (8), 081302-081302-10.

4. $\quad$ Cahill, D. G., Reiew of Scientific Instruments 2004, 75 (12), 5119-5122.

5. Touloukian, Y.; Buyco, E., Specific Heat: Nonmetallic Solids, Vol. 5. Thermophysical Properties of Matter 1970, 19702, 24. 
6. Molgaard, J.; Smeltzer, W., Thermal conductivity of magnetite and hematite. Journal of Applied Physics 1971, 42 (9), 3644-3647.

7. $\quad$ Lide, D. R., CRC handbook of chemistry and physics. CRC press: 2004; Vol. 85.

8. Linnera, J.; Karttunen, A., Ab initio study of the lattice thermal conductivity of $\mathrm{Cu}_{2} \mathrm{O}$ using the generalized gradient approximation and hybrid density functional methods. Physical Review B 2017, 96 (1), 014304.

9. Liu, M. S.; Lin, M. C.; Huang, I. T.; Wang, C. C., Enhancement of thermal conductivity with CuO for nanofluids. Chemical Engineering \& Technology: Industrial Chemistry-Plant Equipment-Process EngineeringBiotechnology 2006, 29 (1), 72-77.

10. Xue, Q.; Xu, W.-M., A model of thermal conductivity of nanofluids with interfacial shells. Materials Chemistry and Physics 2005, 90 (2-3), 298-301.

11. Kusiak, A.; Battaglia, J.-L.; Gomez, S.; Manaud, J.-P.; Lepetitcorps, Y., CuO thin films thermal conductivity and interfacial thermal resistance estimation. The European Physical Journal-Applied Physics 2006, 35 (1), 17-27.

12. Hay, B.; Davee, G.; Filtz, J.-R.; Hameury, J.; Rongione, L., Thermal Conductivity 30: Thermal Expansion 18: Joint Conferences 2009, 461.

13. Marinelli, M.; Mercuri, F.; Zammit, U.; Pizzoferrato, R.; Scudieri, F.; Dadarlat, D., Critical behavior of thermal diffusivity and thermal conductivity of $\mathrm{Cr}_{2} \mathrm{O}_{3}$ at the Neel transition. Physical Review B 1994, 49 (6), 4356-4359. 